Research Paper

\title{
Long-Term Survival and Prognostic Factors in Locoregionally Advanced Nasopharyngeal Carcinoma Patients Treated with TPF Induction Chemotherapy followed by Cisplatin-Combined Concurrent Chemoradiotherapy
}

Qun Zhang $^{1^{*}}$, Yan Wang ${ }^{1 *}$, Jun-Fang Liao ${ }^{*}$, Yu-Feng Ren¹, Guo-Ping Shen ${ }^{1}$, Shao-Qing Niu ${ }^{1}$, Wei Luo ${ }^{2,3,4}{ }^{\bowtie}$

1. Department of Radiotherapy, First affiliated hospital of Sun Yat-sen University, Guangzhou, People's Republic of China

2. Department of Radiotherapy, Sun Yat-sen University Cancer Center, Guangzhou, People's Republic of China

3. State Key Laboratory of Oncology in South China, Guangzhou, People's Republic of China

4. Collaborative Innovation Center for Cancer Medicine, Guangzhou, People's Republic of China

5. Cancer Hospital Chinese Academy of Medical Science, Shenzhen Center, Shenzhen, People's Republic of China

*These authors contribute equally.

$\triangle$ Corresponding author: Dr. Luo, Department of Radiotherapy, Sun Yat-sen University Cancer Center, Guangzhou, 510060, China. Tel/Fax: 86- 13802525907, E-mail address: luowei@sysucc.org.cn

() Ivyspring International Publisher. This is an open access article distributed under the terms of the Creative Commons Attribution (CC BY-NC) license (https://creativecommons.org/licenses/by-nc/4.0/). See http://ivyspring.com/terms for full terms and conditions.

Received: 2018.11.20; Accepted: 2019.05.13; Published: 2019.06.24

\begin{abstract}
Purpose: The objective of this study was to report long-term results of docetaxel, cisplatin, and 5-fluorouracil (TPF) induction chemotherapy followed by concurrent chemoradiotherapy (CCRT) in patients with locoregionally advanced nasopharyngeal carcinoma (NPC) and identify prognostic factors for this group of patients.

Materials and Methods: From December 2010 to January 2015, 109 patients with locoregionally advanced (III-IVB) NPC were included. Patients were scheduled to complete TPF induction chemotherapy followed by cisplatin based CCRT. Failure-free survival (FFS), overall survival (OS), locoregional failure-free survival (LRFFS) and distant failure-free survival (DFFS) served as clinical outcomes. Kaplan-Meier method, Cox proportional hazards model and receiver operating characteristic (ROC) curves were used for analyzing.

Results: With a median follow-up of 60.2 months (range, 7.9-91.6 months), 3-year FFS, OS, LRFFS, and DFFS were $76.8 \%, 85.1 \%, 88.3 \%$, and $84.1 \%$, respectively. The highest incidence rate of recurrence and metastasis were in the first year after treatment. Multivariate analyses showed that age, total time of radiation therapy (RTT), and total time of therapy (TTT) were independent prognostic factors for FFS and OS. Body mass index (BMI), RTT and TTT were significant variables predicting DFFS. TTT was the only independent prognostic factor for LRFFS.

Conclusion: This study indicated that TPF regimen produced encouraging results in Asian patients with locoregionally advanced nasopharyngeal carcinoma. Toxicity was tolerable and reversible. However, overall treatment time is an important factor that we should take into consideration when make plans of induction chemotherapy related treatment.
\end{abstract}

Key words: nasopharyngeal carcinoma, induction chemotherapy, concurrent chemotherapy, intensitymodulated radiotherapy

\section{Introduction}

Nasopharyngeal carcinoma (NPC) is endemic in Southeast Asia, especially in southern China (1). Radiation is the mainstay treatment for non- disseminated NPC. The Intergroup trial 0099 (2) disclosed that a combination of chemotherapy and radiation significantly improve survival in NPC 
patients. From then on, the role of chemotherapy has gradually been clarified by many clinical trials (3-5). Different treatment regimens have been widely used in clinical practice or in clinical trials in order to achieve better efficacy. In addition, with the advent of intensity-modulated radiotherapy (IMRT), locoregional control has increased dramatically, and a $10 \%$ improvement was reported in IMRT group compared to those in groups receiving conventional modality (6-8). Overall survival rate for early stage NPC patients was about $90 \%$ or better (9), but for locoregionally advanced NPC patients, it was still unsatisfying. Approximately $60-70 \%$ of newly diagnosed NPC patients present with stage III-IVB disease (10). Distant metastasis (about 30\%) is the major failure, which remains problematic and ultimately results in death (11). Addition of adjuvant chemotherapy (AC) to concurrent chemotherapy (CCRT) was proved to be useless in reducing metastasis among III-IVB stage NPC patients in a previous study (12). Meanwhile, induction chemotherapy (IC) and concurrent chemotherapy sequence has shown a light on this area. A recent phase 3, multicenter, randomized clinical trial (4) conducted in endemic areas demonstrated that IC regimen with docetaxel, cisplatin and 5-fluorouracil (TPF) benefited distant-failure free survival (DFFS). A significant reduction of distant metastasis in IC group was found when compare to the CCRT group in their study, which translated to improved failure-free survival (FFS) and overall survival (OS). However, the results of long-term survival have not been reported yet.

Thus, this study aimed at reporting long-term survival of a series of locoregionally advanced NPC patients treated with TPF IC followed by CCRT and identifying prognostic factors for this group of patients.

\section{Materials and Methods}

\section{Patients and Treatments}

From December 2010 to January 2015, 109 patients with locoregionally advanced (III-IVB) nasopharyngeal carcinoma treated in San Yat-sun University Cancer Center were included. The 7th edition of the American Joint Committee on Cancer (AJCC) staging system was used for staging, and all patients underwent TPF IC with docetaxel, cisplatin and 5-fluorouracil, followed by IMRT with concomitant chemotherapy.

Patients with previously untreated, non-distant metastatic, newly histologically confirmed nonkeratinising 7th AJCC stage III-IVB nasopharyngeal carcinoma were eligible. The patients has to be 18-69 years with performance status (as per the Eastern
Corporatized Oncology Group) scores of $0-1$, and adequate bone marrow, liver and renal function. Exclusion criteria were as follows: treatment with palliative intent; multiple primary tumors; pregnancy or lactation; a history of previous radiotherapy, chemotherapy or surgery (except diagnostic) to the primary tumor or nodes; any severe coexisting diseases.

The protocol was approved by the ethics committee of Sun Yat-sen University Cancer Center.

\section{Chemotherapy}

All eligible patients received three cycles of IC and two to three cycles of concurrent chemotherapy. IC administered as follows: docetaxel $60 \mathrm{mg} / \mathrm{m}^{2}$ day 1 , cisplatin $65 \mathrm{mg} / \mathrm{m}^{2}$ day 1 and 5-fluorouracil 550 $\mathrm{mg} / \mathrm{m}^{2}$ days $1-5$, repeated every 3 weeks. Concurrent chemotherapy consisted of cisplatin $80 \mathrm{mg} / \mathrm{m}^{2}$ day 1 , repeated every 3 weeks. Dose adjustment of chemotherapy were allowed for following situations: 1. In cases of hematological toxicity, chemotherapy withheld until neutrophil counts and platelet counts recovered to $\geq 1500$ cells per $\mu l$ and $\geq 100000$ cells per $\mu \mathrm{l}$, respectively. 2 . In cases of renal or liver toxicity, chemotherapy withheld until adequate renal function and liver function regained. Dose modification based on nadir blood counts and interim toxicities of preceding cycle. Docetaxel dose reduced by one level $\left(10 \mathrm{mg} / \mathrm{m}^{2}\right)$ if patients presented with following situations: 1 . A second episode of febrile neutropenia, neutropenic infection or neutropenia lasting for longer than 7 days. 2. A first episode of grade 4 thrombocytopenia. 3. Elevation of aspartate aminotransferase, alanine aminotransferase or alkaline phosphatase levels (more than 2.5 to 5.0 times the upper limit of normal). 4. A first episode of grade 4 diarrhea or a second episode of grade 3 diarrhea. 5. A grade 4 mucositis. Cisplatin dose reduced by one level $\left(10 \mathrm{mg} / \mathrm{m}^{2}\right.$ in induction phase and $20 \mathrm{mg} / \mathrm{m}^{2}$ in concurrent phase) if patients had grade 3 neutropenia or grade 2 thrombocytopenia (concurrent phase only), a grade 2 neurotoxicity, creatinine clearance of 40-60 $\mathrm{mL} / \mathrm{min}$ or grade 2 neutropenia. 5 -fluorouracil dose was reduced by one level $\left(100 \mathrm{mg} / \mathrm{m}^{2}\right)$ if patients reported a first episode of grade 3-4 diarrhea or grade 3 mucositis. Chemotherapy was stopped completely in following cases: 1 . Creatinine clearance of $<40$ $\mathrm{mL} / \mathrm{min}$. 2. Aspartate aminotransferase, alanine aminotransferase or alkaline phosphatase $>5$ times the upper limit of normal. 3 . Second episode of grade 4 diarrhea, zgrade 3 neurotoxicity or ototoxicity. Prophylactic granulocyte colony-stimulating factor was only allowed if a patient had febrile neutropenia, a neutropenic infection, a delay in recovery of the absolute neutrophil count at day 28 or grade 4 
neutropenia persisting for $\geq 7$ days in preceding cycle. Prophylactic antibiotics were allowed in grade 4 neutropenia. In addition, chemotherapy stopped if physician in charge believed that further chemotherapy might hamper RT or if patient refused.

\section{Radiotherapy}

Treatment with IMRT was mandatory in this study. Gross tumor volume included primary tumor and enlarged lymph nodes. High-risk clinical target volume was defined as the nasopharyngeal gross tumor volume plus a $5-10 \mathrm{~mm}$ margin $(2-3 \mathrm{~mm}$ posteriorly if adjacent to brainstem or spinal cord) to encompass the high-risk sites of microscopic extension and the whole nasopharynx. Low-risk clinical target volume was defined as high-risk clinical target volume plus a $5-10 \mathrm{~mm}$ margin (2-3 $\mathrm{mm}$ posteriorly if adjacent to brainstem or spinal cord). It included skull base, clivus, sphenoid sinus, parapharyngeal space, pterygoid fossae, posterior parts of the nasal cavity, retropharyngeal nodal regions and the elective neck area from level IB to V. Radiotherapy was performed with 5 fractions $(\mathrm{F})$ weekly of $70 \mathrm{~Gy} /$ $33 \mathrm{~F}$ or $68 \mathrm{~Gy} / 30 \mathrm{~F}$ (2.12 Gy or 2.26 Gy/F). Concurrent chemoradiotherapy (CCRT) administered 3 weeks after start of the last cycle of TPF in intervals.

\section{Evaluation of response and toxicity}

After 2 to 3 weeks completion of last cycle of IC, response to the therapy was assessed by magnetic resonance imaging (MRI) and clinically by flexible nasopharyngoscopy. Treatment response was evaluated according to Response Evaluation Criteria in Solid Tumors criteria (version 1.1) (13) as complete response (CR), partial response (PR), stable disease $(\mathrm{SD})$, disease progression (PD) or not assessable. Effective response was defined as $\mathrm{CR}+\mathrm{PR}$. The Common Toxicity Criteria for Adverse Events (CTCAE V3.0) system (14) was used to evaluate toxicity before each cycle of chemotherapy. Hematologic assessments were performed weekly to determine the toxicity at nadir. The highest grade of toxicity was recorded for analysis in this study.

\section{Follow-up and end-points}

After treatment, patients were followed-up every 3 months for the first 3 years and every 6 months thereafter until the fifth year. The intervals between follow-ups were to 12 months after 5 years. Patients were monitored through phone calls or outpatient clinic follow-up visits. The location and timing of tumor recurrence and metastasis were documented. Patients with locoregional relapse and/or metastatic disease could receive re-irradiation, surgery and/or chemotherapy according to individual situations. Failure-free survival (FFS), overall survival (OS), locoregional failure-free survival (LRFFS) and distant failure-free survival (DFFS) served as clinical outcomes. All intervals were calculated from the start of treatment. OS was defined as the time until death from any cause. FFS was defined as the time until the date of treatment failure or death from any cause, whichever occurred first. LRFFS was defined as the time until the first recurrence in the nasopharynx and/or cervical region after radiotherapy. DFFS was defined as the time until distant metastasis.

\section{Statistical analysis}

Survival rates were calculated using KaplanMeier method. Cox proportional hazards model was used to calculate hazard ratios (HRs). Multivariate analyses were performed using Cox proportional hazards model to test the statistical independent factors. SPSS version 19.0 (SPSS, Inc., an IBM Company; Chicago, IL, USA) was used for statistical analyses. A two-tailed p-value of less than 0.05 was considered as statistically significant.

\section{Results}

\section{Patient characteristics and determination of cutoff values for gap between treatments RTT, and TTT}

A total of 109 patients were included in this study. The last follow-up was in July 30th 2018, median follow-up for the entire cohort was 60.2 months (range, 7.9-91.6 months). Of all the 109 patients, one did not complete radiotherapy because of refusal after a grade 4 neutropenia following a radiation dose of $54.76 \mathrm{~Gy} / 24 \mathrm{~F}$. This patient was included in the toxicity analysis but excluded in survival and prognostic analyses. Characteristics of all patients were listed in Table 1.

Cutoff values for various variables were calculated by using receiver operating characteristic (ROC) curves based on FFS. The cutoff value for age was 46 years (the sensitivity was $78.6 \%$ and the specificity was $55.0 \%)$, with an area of $0.687(\mathrm{P}=0.003)$. Similarly, the optimal cutoff value for the gap period between the initiation of RT and the initiation of IC (GAP $=$ RT initiation date - IC initiation date) was 71 days (the sensitivity was $75.0 \%$ and the specificity was $64.0 \%)$ with an area of $0.640(\mathrm{P}=0.028)$. The optimal cutoff value for RTT (RTT $=$ RT completion date - RT initiation date) was 50 days (the sensitivity was $46.4 \%$ and the specificity was $88.7 \%$ ) with an area of 0.739 (P $<0.001)$. The optimal cutoff value for TTT $($ TTT $=$ all treatment completion date - IC initiation date) was 122 days (the sensitivity was $60.7 \%$ and the specificity was $81.2 \%)$ with an area of $0.674(P=0.006)$. However, 
we could not find a cutoff for BMI, as area under curve was $0.419(\mathrm{P}=0.206)$. Thus, $\mathrm{BMI}$ was analyzed as a continuous variable in multivariate analysis.

\section{Acute and late toxicity}

During IC, grade 3-4 acute toxicities included leukopenia, neutropenia, anemia, hepatic impairment, vomiting and nausea, and diarrhea, which occurred in $57.8 \%, 71.5 \%, 0.9 \%, 0.9 \%, 3.7 \%$, and $11.9 \%$ of cases, respectively. In addition, eight (7.3\%) patients presented with neutropenic fever. During RT period, grade 3-4 toxicities of leukopenia, neutropenia, thrombocytopenia, anemia, vomiting and nausea, diarrhea, dysphagia, and mucositis were found in $17.4 \%, 20.2 \%, 1.8 \%, 0.9 \%, 0.9 \%, 0.9 \%, 10.1 \%$, and $37.6 \%$ of all patients, respectively. Neutropenic fever was recorded in two $(1.8 \%)$ patients. Details of incidence rates of above toxicities were listed in Table 2. In terms of late toxicity, nine $(8.3 \%)$ patients developed unilateral or bilateral hearing loss $(30 \%-50 \%)$, of which three patients gradually developed unilateral deafness. Unilateral tinnitus was detected in one patient. One patient lost sight bilaterally and one lost sight unilaterally. Two patients experienced blurred vision unilaterally. Two patients presented with temporal lobe necrosis, one with masticatory muscle necrosis, and one with dystaxia due to radiation.

\section{Compliance and clinical response}

Of all patients, 108 completed full course of RT and treatment was terminated in one patient due to refusal. Twelve patients $(11.0 \%)$ completed only one cycle of IC, 10 patients received 2 cycles, 87 (79.8\%) patients completed three cycles of IC. In terms of concurrent chemotherapy, only two $(1.8 \%)$ patients received three cycles of cisplatin chemotherapy during RT, as listed in Table 1 . The effective response rate to induction chemotherapy in the nasopharynx was $93.6 \%$ (complete remission $83.5 \%+$ partial remission $10.1 \%), 6.4 \%$ was observed with stable disease. The CR and PR rates were $81.7 \%$ and $14.7 \%$ in the cervical node, while stable disease was in $3.7 \%$ patients in the cervical node (Table 3).

\section{Survival analysis and patterns of disease failure}

Among 108 of 109 patients who completed radiotherapy, 22 (20.4\%) deaths occurred. The 3-year FFS, OS, LRFFS, and DFFS were 76.8\%, 85.1\%, 88.3\%, and $84.1 \%$ respectively. Survival curves of FFS, OS, LRFFS, and DFFS were shown in Figure 1. Twenty-eight patients showed disease progression, 12 $(11.1 \%)$ showed locoregional recurrence and 19 $(17.6 \%)$ developed distant metastases, three patients underwent both, details were shown in Table 4 . In $(11 / 12) \quad 92.0 \%$ of recurrence cases, recurrence occurred in the first three years of follow-up, while only one patient showed disease relapse in the fourth year; no recurrence was found after the fifth year to the last follow-up day. A similar trend was observed in occurrence of metastases. The highest incidence rate of recurrence and metastasis occurred in the first year after treatment (Fig 2).

Table 1. Clinical characteristics of 109 nasopharyngeal carcinoma patients.

\begin{tabular}{|c|c|c|c|}
\hline Characteristic & & Mean & Range \\
\hline Age & year & 45.5 & $18-68$ \\
\hline \multirow[t]{2}{*}{ BMI } & $\mathrm{Kg} / \mathrm{m}^{2}$ & 22.7 & $15.7-32.3$ \\
\hline & & NO. & $\%$ \\
\hline \multirow[t]{2}{*}{ Gender } & Female & 21 & $19.3 \%$ \\
\hline & Male & 88 & $80.7 \%$ \\
\hline \multirow[t]{4}{*}{ T-stage } & 1 & 2 & $1.8 \%$ \\
\hline & 2 & 18 & $16.5 \%$ \\
\hline & 3 & 35 & $32.1 \%$ \\
\hline & 4 & 54 & $49.5 \%$ \\
\hline \multirow[t]{4}{*}{ N-stage } & 0 & 1 & $0.9 \%$ \\
\hline & 1 & 30 & $27.5 \%$ \\
\hline & 2 & 53 & $48.6 \%$ \\
\hline & 3 & 25 & $22.9 \%$ \\
\hline \multirow[t]{3}{*}{ Clinical Stage } & III & 36 & $33.0 \%$ \\
\hline & IVA & 48 & $44.0 \%$ \\
\hline & IVB & 25 & $22.9 \%$ \\
\hline \multirow[t]{3}{*}{ Total Radiation Dose } & 54.8 & 1 & $0.9 \%$ \\
\hline & 68.0 & 13 & $11.9 \%$ \\
\hline & 70.0 & 95 & $87.2 \%$ \\
\hline Cycle of Induction & 1 & 12 & $11.0 \%$ \\
\hline \multirow[t]{2}{*}{ Chemotherapy } & 2 & 10 & $9.2 \%$ \\
\hline & 3 & 87 & $79.8 \%$ \\
\hline Cycle of Concurrent & 2 & 107 & $98.2 \%$ \\
\hline Chemotherapy & 3 & 2 & $1.8 \%$ \\
\hline
\end{tabular}

Abbreviation: $\mathrm{BMI}=$ body mass index, $\mathrm{NO}=$ number, $\mathrm{T}=$ tumor, $\mathrm{N}=$ node.

Table 2. Acute toxicities of the 109 nasopharyngeal carcinoma patients.

\begin{tabular}{|c|c|c|c|c|c|}
\hline \multirow[b]{2}{*}{ Acute toxicity } & \multirow[b]{2}{*}{ Grade } & \multicolumn{2}{|c|}{$\begin{array}{l}\text { Induction } \\
\text { chemotherapy }\end{array}$} & \multicolumn{2}{|c|}{$\begin{array}{l}\text { Concurrent } \\
\text { chemoradiotherapy }\end{array}$} \\
\hline & & NO. & $\%$ & NO. & $\%$ \\
\hline \multirow[t]{2}{*}{ Leukopenia } & 3 & 39 & $35.8 \%$ & 18 & $16.5 \%$ \\
\hline & 4 & 24 & $22.0 \%$ & 1 & $0.9 \%$ \\
\hline \multirow[t]{2}{*}{ Neutropenia } & 3 & 30 & $27.5 \%$ & 15 & $13.8 \%$ \\
\hline & 4 & 48 & $44.0 \%$ & 7 & $6.4 \%$ \\
\hline Neutropenic fever & 1 & 8 & $7.3 \%$ & 2 & $1.8 \%$ \\
\hline \multirow[t]{2}{*}{ Thrombocytopenia } & 3 & 0 & $0.0 \%$ & 1 & $0.9 \%$ \\
\hline & 4 & 0 & $0.0 \%$ & 1 & $0.9 \%$ \\
\hline \multirow[t]{2}{*}{ Anemia } & 3 & 1 & $0.9 \%$ & 1 & $0.9 \%$ \\
\hline & 4 & 0 & $0.0 \%$ & 0 & $0.0 \%$ \\
\hline \multirow[t]{2}{*}{ Hepatic impairment } & 3 & 1 & $0.9 \%$ & 0 & $0.0 \%$ \\
\hline & 4 & 0 & $0.0 \%$ & 0 & $0.0 \%$ \\
\hline Renal impairment & $3-4$ & 0 & $0.0 \%$ & 0 & $0.0 \%$ \\
\hline \multirow[t]{2}{*}{ Vomiting \& Nausea } & 3 & 4 & $3.7 \%$ & 1 & $0.9 \%$ \\
\hline & 4 & 0 & $0.0 \%$ & 0 & $0.0 \%$ \\
\hline \multirow[t]{2}{*}{ Diarrhea1 } & 3 & 6 & $5.5 \%$ & 1 & $0.9 \%$ \\
\hline & 4 & 7 & $6.4 \%$ & 0 & $0.0 \%$ \\
\hline \multirow[t]{2}{*}{ Dysphagia } & 3 & & & 11 & $10.1 \%$ \\
\hline & 4 & & & 0 & $0.0 \%$ \\
\hline Xerostomia & $3-4$ & & & 0 & $0.0 \%$ \\
\hline \multirow[t]{2}{*}{ Mucositis } & 3 & & & 41 & $37.6 \%$ \\
\hline & 4 & & & 0 & $0.0 \%$ \\
\hline
\end{tabular}

Abbreviation: NO. $=$ number. 
Table 3. Clinical response to induction chemotherapy of the 109 nasopharyngeal carcinoma patients.

\begin{tabular}{rlll}
\hline & & NO. & $\%$ \\
\hline Response of Nasopharynx to IC & CR & 91 & $83.5 \%$ \\
& PR & 11 & $10.1 \%$ \\
& SD & 7 & $6.4 \%$ \\
& PD & 0 & $0.0 \%$ \\
Response of Lymph Node to IC & CR & 89 & $81.7 \%$ \\
& PR & 16 & $14.7 \%$ \\
& SD & 4 & $3.7 \%$ \\
& PD & 0 & $0.0 \%$
\end{tabular}

Abbreviation: NO. = number, $\mathrm{IC}=$ induction chemotherapy, $\mathrm{CR}=$ complete remission, $\mathrm{PR}=$ partial remission, $\mathrm{SD}=$ steady disease, $\mathrm{PD}=$ progress disease

Table 4. Mode of treatment failure in the 108 nasopharyngeal carcinoma patients in the study

\begin{tabular}{lll}
\hline Mode of treatment failure & NO. & $\%$ \\
\hline Local only & 5 & $4.6 \%$ \\
Nodal only & 3 & $2.8 \%$ \\
Local + nodal & 1 & $0.9 \%$ \\
Metastasis only & 16 & $14.8 \%$ \\
Local + Metastasis & 1 & $0.9 \%$ \\
Nodal + Metastasis & 2 & $1.9 \%$ \\
\hline
\end{tabular}

Abbreviation: NO. $=$ number.

\section{Univariate analysis}

In univariate analysis, age ( $\geq 46 y$ vs $<46 y)$, N-stage (N2-3 vs. N0-1), GAP ( $\geq 71$ days vs. $<71$ days), RTT ( $\geq 50$ days vs. $<50$ days) and TTT ( $\geq 122$ days vs.
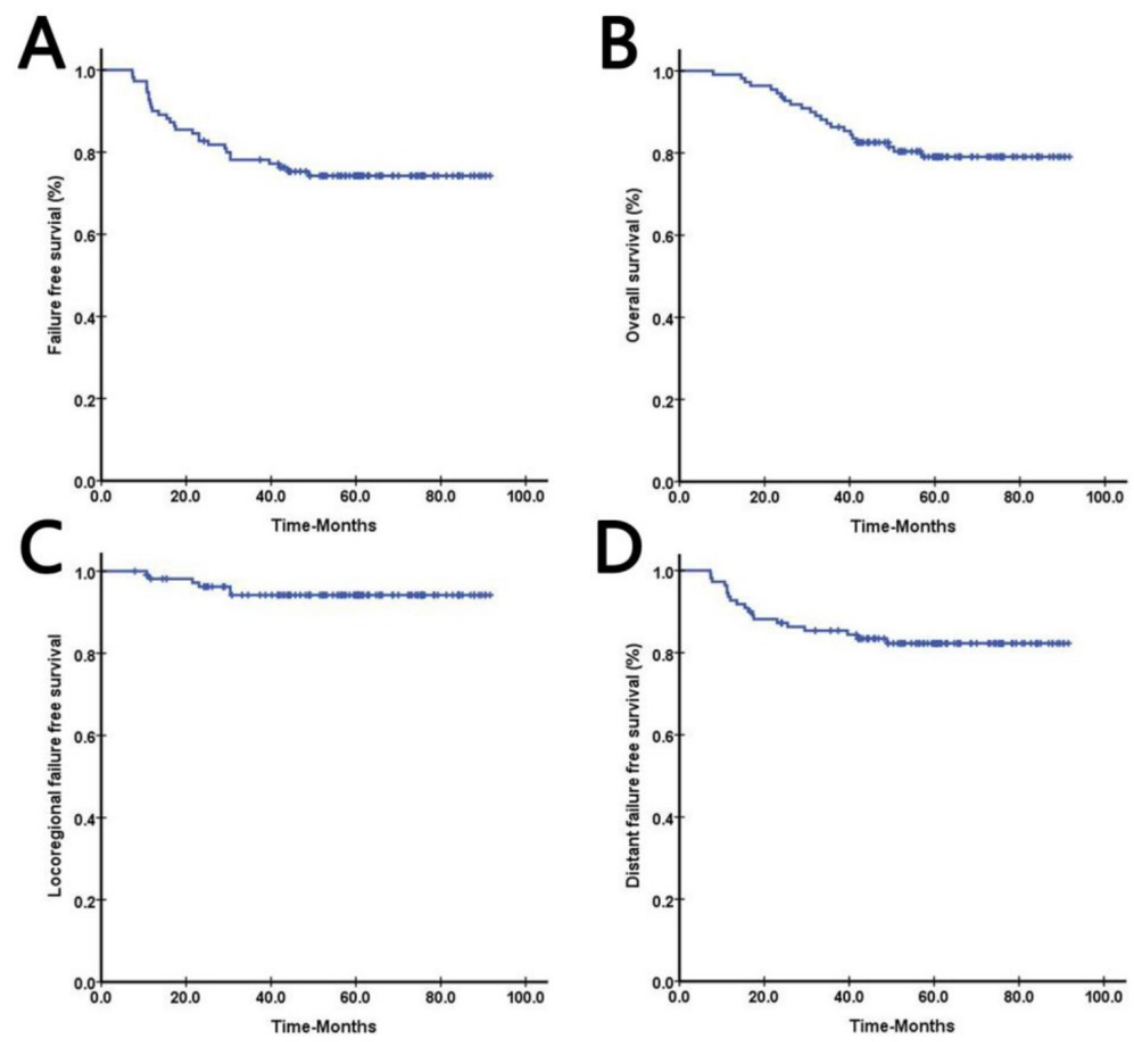

Figure 1. Prognosis of 108 nasopharyngeal carcinoma (NPC) patients: A. Failure-free survival, B. Overall survival, C. Locoregional failure-free survival, D. Distant failure-free survival
$<122$ days) were significant prognostic factors predicting FFS. Age, GAP, RTT and TTT were significant factors predicted OS. GAP, RTT and TTT were significant predictive factors for LRFFS. Age, BMI, N-stage, GAP, RTT and TTT were significant predictive factors for DFFS. Details were listed in Table 5.

\section{Multivariate analysis}

The prognostic factors found in univariate analysis were included in multivariate analysis. As there was a significant correlation between GAP and TTT (correlation coefficient $=0.704, \mathrm{P}<0.001$ ), the variable GAP was not included in multivariate analysis together with TTT. Table 6 summarized the independent predictors for OS, FFS, LRFFS and DFFS. Age, RTT and TTT were independent prognostic factors for OS and FFS ( $\mathrm{P}=0.022,0.001,<0.001$ and $0.007,0.006,<0.001$, respectively). The significant variables that predicted DFFS were BMI, RTT and TTT ( $\mathrm{P}=0.020,0.003$ and 0.012$)$. TTT was the only independent prognostic factor for LRFFS $(\mathrm{P}=0.009)$.

\section{Discussion}

The definition of locoregionally advanced NPC included patients with T3-4 stage and/or N2-3 stage tumors but without systemic metastases according to AJCC staging system (7th edition). It has been reported that locoregionally advanced tumor stage (III-IVB) was an independent risk factor for treatment failure. Distant metastasis has emerged as the predominant manifestation of failure and the key problem to be solved in the new era of highprecision radiotherapy $(6,15)$. From the mid-1990s, CCRT \pm AC has been deemed as standard treatment for locoregionally advanced NPC based on Intergroup 0099 study and three phase III clinical trials in endemic areas (2,16-18). In general, three different strategies have been explored: before (inductive), during (concurrent) and after (adjuvant) RT. Each combined modality has advantages and disadvantages and has been extensively investigated in last two decades. We reviewed a series of articles pertaining to 21 phase II/III randomized trials that investigated the role of CCRT in patients with locoregionally advanced NPC $(2,4,23-32,12,33,16-22)$. 


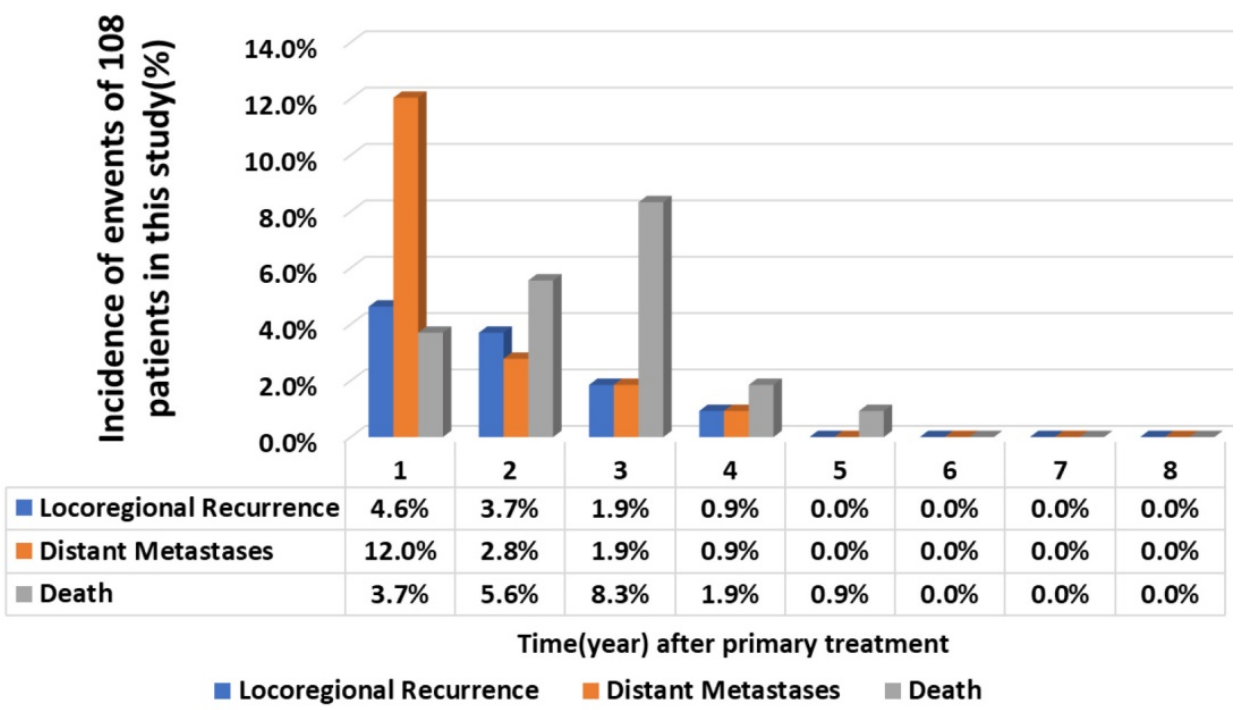

Figure 2. Disease progress trend in 108 nasopharyngeal carcinoma patients.

Table 5. Univariate analysis of other prognostic factors for 108 patients who completed radiation therapy.

\begin{tabular}{|c|c|c|c|c|c|c|c|c|}
\hline & \multicolumn{2}{|l|}{ OS } & \multicolumn{2}{|l|}{ FFS } & \multicolumn{2}{|l|}{ LRFFS } & \multicolumn{2}{|l|}{ DFFS } \\
\hline & HR (95\% CI) & $\mathbf{P}$ & HR (95\% CI) & $\mathbf{P}$ & HR (95\% CI) & $\mathbf{P}$ & HR (95\% CI) & $\mathbf{P}$ \\
\hline \multicolumn{9}{|l|}{ Age } \\
\hline$\geq 46 y$ vs $<46 y$ & $4.284(1.450-12.663)$ & 0.009 & $3.740(1.516-9.230)$ & 0.004 & $2.811(0.761-10.388)$ & 0.121 & $5.269(1.535-18.089)$ & 0.008 \\
\hline \multicolumn{9}{|l|}{ Gender } \\
\hline Male vs. Female & $1.553(0.459-5.250)$ & 0.479 & $2.091(0.631-6.927)$ & 0.227 & $1.220(0.267-5.570)$ & 0.797 & $2.062(0.476-8.928)$ & 0.333 \\
\hline BMI & $0.881(0.768-1.011)$ & 0.072 & $0.907(0.803-1.024)$ & 0.115 & $0.976(0.814-1.169)$ & 0.791 & $0.845(0.728-0.981)$ & 0.027 \\
\hline \multicolumn{9}{|l|}{ T-stage } \\
\hline T3-4 vs. T1-2 & $0.538(0.210-1.376)$ & 0.196 & $0.593(0.252-1.395)$ & 0.231 & $1.013(0.222-4.628)$ & 0.986 & $0.434(0.165-1.144)$ & 0.091 \\
\hline \multicolumn{9}{|l|}{ N-stage } \\
\hline N3 vs. N0-2 & $2.182(0.913-5.215)$ & 0.079 & 2.544 (1.189-5.447) & 0.016 & $1.876(0.564-6.237)$ & 0.304 & $2.688(1.077-6.708)$ & 0.034 \\
\hline \multicolumn{9}{|l|}{ Clinical Stage } \\
\hline IV vs. III & $1.870(0.689-5.074)$ & 0.219 & $2.522(0.958-6.639)$ & 0.061 & $5.995(0.774-46.442)$ & 0.086 & $1.980(0.657-5.969)$ & 0.225 \\
\hline \multicolumn{9}{|l|}{ Gap } \\
\hline$\geq 71 \mathrm{D}$ vs. $<71 \mathrm{D}$ & $6.555(2.207-19.468)$ & 0.001 & $4.349(1.845-10.250)$ & 0.001 & $4.036(1.091-14.925)$ & 0.037 & $3.813(1.370-10.613)$ & 0.010 \\
\hline \multicolumn{9}{|l|}{ RTT } \\
\hline$\geq 50 \mathrm{D}$ vs. $<50 \mathrm{D}$ & $6.989(3.003-16.263)$ & $<0.001$ & $5.245(2.483-11.079)$ & $<0.001$ & $3.711(1.171-11.756)$ & 0.026 & $6.219(2.511-15.404)$ & $<0.001$ \\
\hline \multicolumn{9}{|l|}{ TTT } \\
\hline$\geq 122 \mathrm{D}$ vs. $<122 \mathrm{D}$ & $6.141(2.533-14.885)$ & $<0.001$ & $5.260(2.449-11.296)$ & $<0.001$ & $6.281(1.881-20.974)$ & 0.003 & $4.174(1.667-10.453)$ & 0.002 \\
\hline \multicolumn{9}{|l|}{ Total Dose } \\
\hline 70Gy vs. 68Gy & $0.980(0.290-3.315)$ & 0.974 & $1.233(0.372-4.084)$ & 0.732 & $1.624(0.210-12.583)$ & 0.642 & $0.765(0.223-2.626)$ & 0.670 \\
\hline
\end{tabular}

Table 6. Significant predictors for long-term survival by multivariate analysis.

\begin{tabular}{|c|c|c|c|c|}
\hline & Significant factors & Hazard Ratio & $95 \% \mathrm{CI}$ & $\mathbf{P}$ \\
\hline \multirow[t]{3}{*}{ OS } & Age ( $\geq 46$ y vs $<46 y)$ & 3.603 & $1.204-10.785$ & 0.022 \\
\hline & $\operatorname{RTT}(\geq 50 \mathrm{D}$ vs. $<50 \mathrm{D})$ & 4.551 & $1.901-10.899$ & 0.001 \\
\hline & $\mathrm{TTT}(\geq 122 \mathrm{D}$ vs. $<122 \mathrm{D})$ & 5.414 & $2.157-13.591$ & $<0.001$ \\
\hline \multirow[t]{3}{*}{ FFS } & Age ( $\geq 46$ y vs $<46 y)$ & 3.546 & $1.419-8.865$ & 0.007 \\
\hline & RTT( $\geq 50 \mathrm{D}$ vs. $<50 \mathrm{D})$ & 3.137 & $1.388-7.093$ & 0.006 \\
\hline & $\operatorname{TTT}(\geq 122$ D vs $<122$ D) & 4.532 & $2.025-10.144$ & $<0.001$ \\
\hline LRFFS & TTT( $\geq 122 \mathrm{D}$ vs. $<122 \mathrm{D})$ & 5.244 & $1.518-18.115$ & 0.009 \\
\hline \multirow[t]{3}{*}{ DFFS } & BMI & 0.831 & $0.711-0.971$ & 0.020 \\
\hline & RTT( $\geq 50 \mathrm{D}$ vs. $<50 \mathrm{D})$ & 4.408 & $1.641-11.838$ & 0.003 \\
\hline & $\mathrm{TTT}(\geq 122 \mathrm{D}$ vs. $<122 \mathrm{D})$ & 3.406 & $1.314-8.826$ & 0.012 \\
\hline
\end{tabular}

Abbreviation: OS $=$ overall survival, FFS $=$ failure free survival, LRFFS $=$ locoregional failure-free survival, DFFS $=$ distant failure-free survival, $\mathrm{RTT}=$ radiation therapy completion date - radiation therapy initiation date, TTT $=$ all treatments completion date - induction chemotherapy initiation date.
In 1998, the Intergroup trial 0099 based in the United Stated (2) was terminated prematurely, as the interim analysis disclosed a significant survival advantage favoring the combined concurrentadjuvant chemoradiotherapy group (OS increase of about $31 \%$ in this group when compared to RT alone). However, a series of concerns were raised as follows: 1. The inferior results of RT-alone group were not matched with that of the counterpart group in the endemic area. 2. The proportion of NPC patients with non-keratinizing undifferentiated carcinoma (World Health Organization [WHO] types III) was only $41 \%$ in this study, which was more common in endemic regions (34). Whether the results could be directly 
applied in non-American NPC patients was uncertain. According to result of 0099, CCRT + AC had been recommended as the standard treatment for locoregionally advanced NPC by National Comprehensive Cancer Network (NCCN) for a long time. Three additional trials $(16,19,20)$ were conducted in Asia to further confirm the efficacy and safety of this treatment modality. These trials found no significant difference in OS rates between CCRT + AC group and RT alone group. Further, a different trial (19) revealed a concerning increase in non-cancer deaths in the CCRT + AC group: $1.7 \%$ treatmentrelated mortalities and $4.7 \%$ were because of "incidental" causes (including infection, second malignancy and suicide). And the compliance of AC reported in the above studies was low. Contemporaneously, studies evaluating IC + RT vs. RT were conducted in southern China. IC, regarded as a promising strategy, was administered with an aim to eradicate distant micro-metastases and decrease tumor burden at the primary site before radiation. However, five phase III trials (25-29) demonstrated accordant results that IC failed to achieve a significant survival advantage in locoregionally advanced NPC. Researchers of these studies speculated that the reasons for the negative results might as follows: 1 . Agents and dose administered might not been adequate. 2. Two to three cycles of IC may not sufficient to eradicate all distant micro-metastases. 3 . Screening criteria included more patients with low risk for distant failure. 4. Prevalence of an excess of chemotherapy-related deaths and RT refusal. Three other trials have tested RT alone + AC regimen (21-23) and consistently found no survival advantage from AC after RT alone. A lower OS in RT + AC group was observed, although no significant difference was found. Taken together, we cannot exclude the possibility that subtle damage by AC may have led to the observed non-cancer-related deaths. Thereafter, studies focusing on CCRT aimed to find optimal treatment modes for NPC. Phase III trials of CCRT vs. RT alone conducted in Taiwan (18), Hong Kong (17), and Guangzhou (24) found satisfactory results favoring the CCRT group. In the year 2012, one study with a large simple size conducted by Chen et al. showed that the addition of AC to CCRT did not significantly improve survival compared with CCRT alone (12). The NCCN Panel revised the recommendation from category 1 to category $2 \mathrm{~A}$ of CCRT + AC for NPC. Therefore, doctors have shown renewed interest in re-exploration of "inductiveconcurrent" strategy in locoregionally advanced NPC. The results of a phase II randomized trial (31) suggested that inductive docetaxel and cisplatin chemotherapy followed by cisplatin combined with
RT was a highly feasible sequential strategy for locoregionally advanced NPC. The preliminary results of this trial showed $10 \%$ reduction of distant metastases by using this regimen. However, a phase III randomized study (32) conducted in Singapore failed to show improved survival by adding IC to concurrent chemotherapy. And another study (33) from Guangzhou demonstrated superior DFS but not OS. Apart from reasons mentioned previously, the following two reasons were considered in these studies: 1 . The use of IMRT could have led to better disease control in both arms, and may have impinged on the efficacy of IC. 2. The possibility of accelerated repopulation. Nevertheless, it was recognized that more effective IC regimens may well exist. Recently, one study conducted in Guangzhou (4) reported that the most suitable IC regimen for locoregionally advanced NPC patients with more rigorous inclusion criteria of stage III-IVB and excluding stages T3-4N0 was TPF in three cycles, every 3 weeks. The 3 -year FFS and $\mathrm{OS}$ were $80 \%$ vs. $72 \%(\mathrm{P}=0.034)$ and $92 \%$ vs. $86 \%$ $(\mathrm{P}=0.029)$ in the IC + CCRT group and the CCRT group, respectively. IC followed by $\mathrm{RT} \pm$ chemotherapy was revised as a category $2 \mathrm{~A}$ recommendation in NCCN guideline based on this study.

In our study, the 3-year FFS was in agreement with that reported in previous studies $(4,32,33)$ ( $76.80 \%$ in ours vs. $75 \%, 80 \%, 82 \%$ in previous studies) and was slightly improved when compared with historical controls that used CCRT alone $(67.4 \%, 72 \%$, $74.1 \%)$. The OS was slightly lower than that reported by above studies $(85.1 \%$ in the present study vs. $94 \%$, $92 \%$, and $88.5 \%$ in previous studies). More than half of patients experienced delays ( $\geq 110$ days, the planned treatment time interval) in the scheduled treatment plan. The most common factors that contributed to the high percentage of delays in administration of RT were myelosuppression and acute toxicity during chemoradiotherapy (especially oral mucositis). One of the most important finding in present study was that RTT and TTT were independent prognostic factors for FFS, OS and DFFS. In contrast to findings of current study, one previous study showed that in patients who underwent CCRT alone, during the total treatment time between 36 to 63 days, a mild delay did not compromise tumor control and OS (35). The primary difference between these two studies lies in the use of IC. Therefore, we speculated that accelerated repopulation triggered by IC was thought to be the possible reason for the difference in outcomes (36-38). If so, the subsequent RT may render less effective when it was administered at the time of accelerated tumor repopulation. Moreover, this hypothesis could explain why impressive initial tumor response to IC did not translate into enhanced 
tumor control in several early studies $(32,39)$. Concurrent chemotherapy after IC could be one way to counter this negative effect of IC. The use of more cycles in the inductive setting needs to be re-examined since this type of regimen may allow accelerated repopulation of surviving tumor cells and impair local control that could have be achieved by subsequent RT.

The agents, dosage, course, timing of delivery chemotherapy and screening criteria for NPC patients are still controversial. The primary focus of current clinical trials includes therapeutic agents, treatment course, and inclusion criteria. Ongoing trials such as the NCT01872962 and NCT02512315 aim to address the role of induction chemotherapy and find individualized treatments for patients with locoregionally advanced NPC.

In conclusion, this study indicated that TPF regimen produced encouraging results in Asian patients with locoregionally advanced nasopharyngeal carcinoma. Toxicity was tolerable and reversible. However, overall treatment time is an important factor that we should take into consideration when make plans of induction chemotherapy related treatment.

\section{Competing Interests}

The authors have declared that no competing interest exists.

\section{References}

1. Torre LA, Bray F, Siegel RL, Ferlay J, Lortet-Tieulent J, Jemal A. Global cancer statistics, 2012. CA Cancer J Clin. 2015 Mar;65(2):87-108.

2. Al-sarraf BM, Leblanc M, Giri PGS, Fu KK, Cooper J, Vuong T, et al. Chemoradiotherapy Versus Radiotherapy in Patients With Advanced Nasopharyngeal Cancer: Phase III Randomized Intergroup Study 0099. Society. 1998;16(4):1310-7.

3. Yang J, Pan P, Song W, Huang R, Li J, Chen K, et al. Voxelwise meta-analysis of gray matter anomalies in Alzheimer's disease and mild cognitive impairment using anatomic likelihood estimation. J Neurol Sci [Internet]. 2012;316(1-2):21-9. Available from: http://dx.doi.org/10.1016/j.jns.2012.02. 010

4. Sun Y, Li WF, Chen NY, Zhang N, Hu GQ, Xie FY, et al. Induction chemotherapy plus concurrent chemoradiotherapy versus concurrent chemoradiotherapy alone in locoregionally advanced nasopharyngeal carcinoma: a phase 3, multicentre, randomised controlled trial. Lancet Oncol [Internet]. 2016;17(11):1509-20.

5. Wee J, Tan EH, Tai BC, Wong HB, Leong SS, Tan T, et al. Randomized trial of radiotherapy versus concurrent chemoradiotherapy followed by adjuvant chemotherapy in patients with American Joint Committee on Cancer/ International Union Against Cancer stage III and IV nasopharyngeal cancer of the endemic variety. J Clin Oncol. 2005;23(27):6730-8.

6. Liu X, Tang LL, Du XJ, Li WF, Chen L, Zhou GQ, et al. Changes in disease failure risk of nasopharyngeal carcinoma over time: Analysis of 749 patients with long-term follow-up. J Cancer. 2017;8(3):455-9.

7. Peng G, Wang T, Yang KY, Zhang S, Zhang T, Li Q, et al. A prospective, randomized study comparing outcomes and toxicities of intensity-modulated radiotherapy vs. conventional two-dimensional radiotherapy for the treatment of nasopharyngeal carcinoma. Radiother Oncol [Internet]. 2012;104(3):286-93. Available from: http://dx.doi.org/10.1016/j.radonc.2012. 08.013

8. Zhang M-X, Li J, Shen G-P, Zou X, Xu J-J, Jiang R, et al. Intensity-modulated radiotherapy prolongs the survival of patients with nasopharyngeal carcinoma compared with conventional two-dimensional radiotherapy: A 10-year experience with a large cohort and long follow-up. Eur J Cancer [Internet]. 2015;51(17):2587-95. Available from: http://dx.doi.org/10.1016/ j.ejca.2015.08.006
9. Su Z, Mao YP, Tang J, Lan XW, OuYang PY, Xie FY. Long-term outcomes of concurrent chemoradiotherapy versus radiotherapy alone in stage II nasopharyngeal carcinoma treated with IMRT: a retrospective study. Tumor Biol. 2016;37(4):4429-38.

10. Mao Y-P, Xie F-Y, Liu L-Z, Sun Y, Li L, Tang L-L, et al. Re-evaluation of 6th edition of AJCC staging system for nasopharyngeal carcinoma and proposed improvement based on magnetic resonance imaging. Int J Radiat Oncol Biol Phys. 2009 Apr;73(5):1326-34

11. Wee J. Nasopharyngeal cancer: a promising future. Lancet Oncol. 2012 Feb;13(2):116-8.

12. Chen L, Hu C-S, Chen X-Z, Hu G-Q, Cheng Z-B, Sun Y, et al. Concurrent chemoradiotherapy plus adjuvant chemotherapy versus concurrent chemoradiotherapy alone in patients with locoregionally advanced nasopharyngeal carcinoma: a phase 3 multicentre randomised controlled trial. Lancet Oncol [Internet]. 2012;13(2):163-71. Available from: http://linkinghub.elsevier.com/retrieve/pii/S1470204511703205

13. Watanabe H, Okada M, Kaji Y, Satouchi M, Sato Y, Yamabe Y, et al. New response evaluation criteria in solid tumours-revised RECIST guideline (version 1.1). Gan To Kagaku Ryoho. 2009 Dec;36(13):2495-501.

14. Trotti A, Colevas AD, Setser A, Rusch V, Jaques D, Budach V, et al. CTCAE v3.0: development of a comprehensive grading system for the adverse effects of cancer treatment. Semin Radiat Oncol. 2003 Jul;13(3):176-81.

15. Am MIKMK, Eo PEMLT, Hau RIMCC, Heung KYC, Hoi PEHKC, Wan WHK, et al. Treatment of Nasopharyngeal Carcinoma with Intensity Modulated Radiotherapy: The HONG KONG Experience. 2004;60(5):1440-50.

16. Wee J, Tan EH, Tai BC, Wong HB, Leong SS, Tan T, et al. Randomized trial of radiotherapy versus concurrent chemoradiotherapy followed by adjuvant chemotherapy in patients with American Joint Committee on Cancer/International Union Against Cancer stage III and IV nasopharyngeal cancer of the endemic variety. J Clin Oncol. 2005;23(27):6730-8.

17. Chan ATC, Leung SF, Ngan RKC, Teo PML, Lau WH, Kwan WH, et al. Overall survival after concurrent cisplatin-radiotherapy compared with radiotherapy alone in locoregionally advanced nasopharyngeal carcinoma. J Natl Cancer Inst. 2005;97(7):536-9.

18. Lin JC, Jan JS, Hsu CY, Liang WM, Jiang RS, Wang WY. Phase III study of concurrent chemoradiotherapy versus radiotherapy alone for advanced nasopharyngeal carcinoma: Positive effect on overall and progression-free survival. J Clin Oncol. 2003;21(4):631-7.

19. Lee AWM, Tung SY, Chua DTT, Ngan RKC, Chappell R, Tung R, et al. Randomized trial of radiotherapy plus concurrent-adjuvant chemotherapy vs radiotherapy alone for regionally advanced nasopharyngeal carcinoma. J Natl Cancer Inst. 2010;102(15):1188-98.

20. Chen Y, Sun Y, Liang S-B, Zong J-F, Li W-F, Chen M, et al. Progress report of a randomized trial comparing long-term survival and late toxicity of concurrent chemoradiotherapy with adjuvant chemotherapy versus radiotherapy alone in patients with stage III to IVB nasopharyngeal carcinoma from endemic regions of Ch. Cancer [Internet]. 2013;119(12):2230-8. Available from: http://doi.wiley.com/10.1002/cncr.28049

21. Rossi A, Molinari R, Boracchi P, Del Vecchio M, Marubini E, Nava M, et al. Adjuvant chemotherapy with vincristine, cyclophosphamide, and doxorubicin after radiotherapy in local-regional nasopharyngeal cancer: Results of a 4-year multicenter randomized study. J Clin Oncol. 1988;6(9):1401-10.

22. Chi K-H, Chang Y-C, Guo W-Y, Leung M-J, Shiau C-Y, Chen S-Y, et al. A phase III study of adjuvant chemotherapy in advanced nasopharyngeal carcinoma patients. Int J Radiat Oncol Biol Phys. 2002;52(5):1238-44.

23. Kwong DLW, Sham JST, Au GKH, Chua DTT, Kwong PWK, Cheng ACK, et al. Concurrent and adjuvant chemotherapy for nasopharyngeal carcinoma: A factorial study. J Clin Oncol. 2004;22(13):2643-53.

24. Zhang L, Zhao C, Peng PJ, Lu LX, Huang PY, Han F, et al. Phase III study comparing standard radiotherapy with or without weekly oxaliplatin in treatment of locoregionally advanced nasopharyngeal carcinoma: Preliminary results. J Clin Oncol. 2005;23(33):8461-8

25. Chan ATC, Teo PML, Leung TWT, Leung SF, Lee WY, Yeo W, et al. A prospective randomized study of chemotherapy adjunctive to definitive radiotherapy in advanced nasopharyngeal carcinoma. Int J Radiat Oncol Biol Phys. 1995;33(3):569-77.

26. Cvitkovic E, Eschwege F, Rahal M, Dosen, Mersic Z, Krajina Z, et al. Preliminary results of a randomized trial comparing neoadjuvant chemotherapy (cisplatin, epirubicin, bleomycin) plus radiotherapy vs. Radiotherapy alone in stage IV $(\leq \mathrm{N} 2, \mathrm{M} 0)$ undifferentiated nasopharyngeal carcinoma: A positive effect on progression-free. Int J Radiat Oncol Biol Phys. 1996;35(3):463-9.

27. Chua DTT, Sham JST, Choy D, Lorvidhaya V, Sumitsawan Y, Thongprasert S, et al. Preliminary report of the Asian-Oceanian Clinical Oncology Association randomized trial comparing cisplatin and epirubicin followed by radiotherapy versus radiotherapy alone in the treatment of patients with locoregionally advanced nasopharyngeal carcinom. Cancer. 1998;83(11):227083.

28. Ma J, Mai H-Q, Hong M-H, Min H-Q, Mao Z-D, Cui N-J, et al. Results of a Prospective Randomized Trial Comparing Neoadjuvant Chemotherapy Plus Radiotherapy With Radiotherapy Alone in Patients With Locoregionally Advanced Nasopharyngeal Carcinoma. J Clin Oncol [Internet]. 2001;19(5): 1350-7. Available from: http://ascopubs.org/doi/10.1200/JCO.2001.19.5.1350 
29. Hareyama M, Sakata K, Shirato H, Nishioka T, Nishio M, Suzuki K, et al. A prospective, randomized trial comparing neoadjuvant chemotherapy with radiotherapy alone in patients with advanced nasopharyngeal carcinoma. Cancer. 2002;94:2217-23.

30. Huang P, Cao K, Guo X, Mo H, Guo L, Xiang Y. A randomized trial of induction chemotherapy plus concurrent chemoradiotherapy versus induction chemotherapy plus radiotherapy for locoregionally advanced nasopharyngeal carcinoma. Oral Oncol. 2012;48:1038-44.

31. Hui EP, Ma BB, Leung SF, King AD, Mo F, Kam MK, et al. Randomized phase II trial of concurrent cisplatin-radiotherapy with or without neoadjuvant docetaxel and cisplatin in advanced nasopharyngeal carcinoma. J Clin Oncol. 2009;27(2):242-9.

32. Tan T, Lim WT, Fong KW, Cheah SL, Soong YL, Ang MK, et al. Concurrent chemo-radiation with or without induction gemcitabine, carboplatin, and paclitaxel: A randomized, phase 2/3 trial in locally advanced nasopharyngeal carcinoma. Int J Radiat Oncol Biol Phys [Internet]. 2015;91(5):952-60. Available from: http://dx.doi.org/10.1016/j.ijrobp.2015.01.002

33. Cao SM, Yang Q, Guo L, Mai HQ, Mo HY, Cao KJ, et al. Neoadjuvant chemotherapy followed by concurrent chemoradiotherapy versus concurrent chemoradiotherapy alone in locoregionally advanced nasopharyngeal carcinoma: A phase III multicentre randomised controlled trial. Eur J Cancer [Internet]. 2017;75:14-23. Available from: http://dx.doi.org/10.1016/j.ejca. 2016.12.039

34. Chua MLK, Wee JTS, Hui EP, Chan ATC. Nasopharyngeal carcinoma. Lancet [Internet]. 2015;6736(15):1-13.

35. Li P, Jin T, Luo D, Shen T, Mai D, Hu W, et al. Effect of Prolonged Radiotherapy Treatment Time on Survival Outcomes after IntensityModulated Radiation Therapy in Nasopharyngeal Carcinoma. PLoS One [Internet]. 2015;10(10):e0141332. Available from: http://journals.plos.org/ plosone/article?id=10.1371/journal.pone. 0141332

36. Tannock IF. Combined modality treatment with radiotherapy and chemotherapy. Radiother Oncol. 1989;16(2):83-101.

37. Davis AJ, Tannock IF. Repopulation of tumour cells between cycles of chemotherapy : a neglected factor. Lancet Oncol. 2000;1(October):86-93.

38. Bourhis J, Wilson G, Wibault P, Janot F, Bosq J, Armand JP, et al. Rapid tumor cell proliferation after induction chemotherapy in oropharyngeal cancer. Laryngoscope. 1994 Apr;104(4):468-72.

39. Huang PY, Zeng $\mathrm{Q}$, Cao KJ, Guo X, Guo L, Mo HY, et al. Ten-year outcomes of a randomised trial for locoregionally advanced nasopharyngeal carcinoma: A single-institution experience from an endemic area. Eur J Cancer [Internet]. 2015;51(13):1760-70. Available from: http://dx.doi.org/10.1016/j.ejca.2015. 05.025 